\title{
ANALYSIS OF THE DIVERSITY POTENTIAL OF AN INTELLIGENT QUADRIFILAR HELIX ANTENNA
}

\author{
T W C Brown, K C D Chew and S R Saunders
}

Centre for Communication Systems Research, University of Surrey, UK

Antenna diversity has been used for many years as a method to mitigate multipath fading. For mobile communications, this has usually been conventionally at the base station, although there is an increasing demand for diversity antennas to be implemented at the mobile. The most common occurrence of this is in the Personal Digital Communications (PDC) network deployed in the Far East (1). Mobile handsets designed for this network often consist of a main antenna, like any other handset and also a smaller diversity antenna. Such a combination gives scope for a dual branch diversity system consisting of some spatial, angular and polarisation diversity (2). While such antennas may provide two signals with low correlation and similar signal to noise ratio, as required for diversity, it is difficult for them both to maintain high Mean Effective Gain (MEG), which is a disadvantage to the overall performance of the system. Further to this, implementing more than two elements is extremely difficult without increasing the handset volume, thereby limiting the maximum diversity gain achievable.

An Intelligent Quadrifilar Helix Antenna (IQHA) (3) is based on four helix antenna elements that can be combined as such to allow beam steering towards satellite and terrestrial base stations. Combining the helix elements in an appropriate fashion gives scope for diversity, such that measurements and simulations have shown the IQHA has potential for up to $23 \mathrm{~dB}$ diversity gain (or system gain as will be seen) in a Rayleigh fading environment (4). Essentially the IQHA is a fourbranch diversity system.

This paper investigates why an IQHA provides such good diversity potential using appropriate analysis techniques (2) and reasonable assumptions for the relevant propagation parameters. As will be seen, an IQHA is primarily an angular diversity system and it is also shown that the Equal Gain Combining (EGC) method (5) provides the highest system gain since there is a significant increase in MEG. This analysis was carried out both for the standard sized IQHA and the reduced-size meandered IQHA (6).

\section{PROPAGATION ENVIRONMENT}

To evaluate the diversity, two key parameters need to be decided upon, namely the angle of arrival and the crosspolar ratio, XPR. These two parameters will therefore be considered separately.

\section{Angle of Arrival}

The Angle of Arrival (AOA) at the mobile is still under investigation to a degree at a number of frequencies and locations. In the general case, for an urban environment (as will be considered in this paper), the azimuth AOA is generally uniform (2) since there is equal chance of a scatterer being at any angle. For elevation AOA it is not so simple. The varying building heights amongst other factors will strongly influence this, although the incoming angles are normally between $0^{\circ}$ and $40^{\circ}$ above the horizontal. An appropriate model is that of Taga (7), which assumes Gaussian elevation AOA with around $20^{\circ}$ mean and $20^{\circ}$ standard deviation so this will be used for analysis here.

\section{Cross Polar Ratio}

The cross-polar ratio, $X P R$, at the mobile varies significantly between different locations and frequencies (2). For this paper $6 \mathrm{~dB}$ will be assumed as appropriate for an urban environment although this has to be considered as variable, which could potentially influence the diversity if investigated in greater detail.

\section{ANTENNA SPECIFICATIONS}

The antennas analysed were a standard 2GHz IQHA (3) and also a reduced sized meandered IQHA (6) also operating at $2 \mathrm{GHz}$. In terms of size and dimensions, the standard IQHA (shown in Figure 1 (a)) was $88 \mathrm{~mm}$ in length and $14 \mathrm{~mm}$ in diameter. The IQHA tracks were printed onto flexible printed circuit board $95 \mathrm{~mm}$ in length with track width $2 \mathrm{~mm}$ spaced $7 \mathrm{~mm}$ apart. The IQHA was formed by rolling the PCB into a cylinder (3). Each port was fed with quarter wavelength semi rigid cables and an SMA connector.

The same construction method was applied to the meandered IQHA, which has considerably reduced dimensions for similar performance as shown in Figure 1 (b). The axial length was reduced to $45 \mathrm{~mm}$ with the same $14 \mathrm{~mm}$ diameter. The lines on the IQHA were meandered by 5 turns with a track spacing of $4 \mathrm{~mm}$. The total track length was $144 \mathrm{~mm}$ to account for the inherent parasitic reactances. 

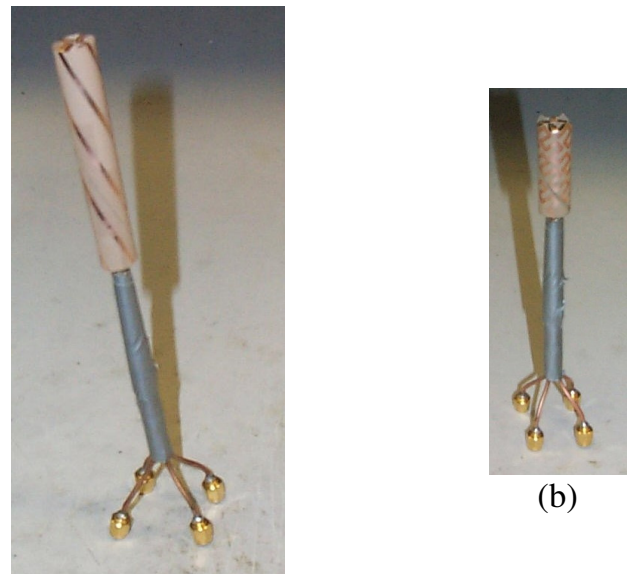

(b)

(a)

Figure 1 Photographs showing (a) the standard 2GHz IQHA and (b) the reduced sized meandered IQHA

\section{DIVERSITY ANALYSIS}

To evaluate the diversity performance it is necessary to consider the correlation of the four branches, their MEG (as well as the MEG of any combined patterns) and the resulting system gain. It must be noted that there is an important difference between the diversity gain and the overall system gain as will be explained.

\section{Correlation}

For an IQHA, it is possible to evaluate the spatial, polarisation, and angular correlation to identify the different contributions to diversity (2). However, it is noted that an IQHA has its four feed points close together such that the phase centres of the four antenna elements are so close (within $4 \mathrm{~mm}$ ) they can be assumed co-located. Therefore, in this application there is no scope for spatial diversity in the strict sense, since the antenna elements are not spaced apart significantly compared to the wavelength. Consequently only the angular correlation and the polarisation correlation (which is inherent within angular correlation (2)) need to be evaluated. The complex angular correlation, $\rho_{12 A}$, for branches 1 and 2 of an IQHA is defined by the following equation (2):

$$
\rho_{12 A}=\frac{\int_{0}^{\pi} \int_{0}^{2 \pi}\left(X P R E_{\theta 1}(\theta, \phi) E_{\theta 2}^{*}(\theta, \phi) p_{\theta}(\theta, \phi)+E_{\phi 1} E_{\phi 2}^{*} p_{\phi}(\theta, \phi)\right) \sin \theta d \phi d \theta}{\mid \int_{0}^{\pi} \int_{-\pi}^{2 \pi}\left(X P R\left|E_{\theta 1}(\theta, \phi)\right|^{2} p_{\theta}(\theta, \phi)+\left|E_{\phi 2}(\theta, \phi)\right|^{2} p_{\phi}(\theta, \phi)\right) \sin \theta d \phi d \theta}
$$

where the antenna patterns for the first element are in two polarisations, $E_{\theta 1}(\theta, \phi)$ and $E_{\phi 1}(\theta, \phi)$. The same applies to the second branch. The AOA is defined by probability density functions, $p_{\theta}(\theta, \phi)$ and $p_{\phi}(\theta, \phi)$ for both polarisations. All of these quantities are functions of azimuth angle, $\phi$, and elevation angle, $\theta$.
The angular correlation is essentially the same as the overall correlation, since there is no spatial decorrelation. Another point to note is that the correlation between the other branches can be evaluated in the same way. However, for a vertical IQHA in an urban fading environment it is the case that $\rho_{12 A}=\rho_{14 A}=\rho_{21 A}=\rho_{23 A}=$ $\rho_{32 A}=\rho_{34 A}$ and $\rho_{24 A}=\rho_{42 A}=\rho_{13 A}=\rho_{31 A}$ if the feed points are numbered as in Figure 2. Therefore, since the diversity of a vertical IQHA will be analysed, only $\rho_{12 A}$ and $\rho_{13 A}$ need be considered (i.e. adjacent and opposite elements).

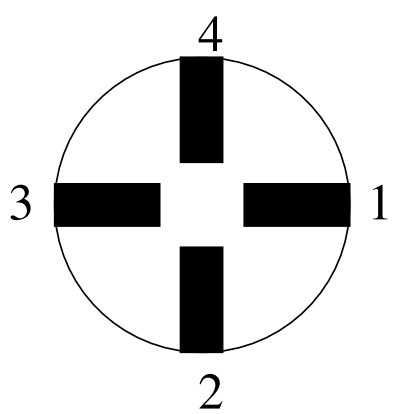

Figure 2 Numbering of IQHA input feed points

The polarisation correlation can be evaluated by considering the relative polarisations of the branches. It must be noted that this is carried out by comparing the relative $E_{\theta}$ and $E_{\phi}$ polarisations rather than the vertical and horizontal (i.e. $E_{x}$ and $E_{y}$ ) polarisations. The equation used to derive the polarisation correlation, $\rho_{12 P}$, is as follows:

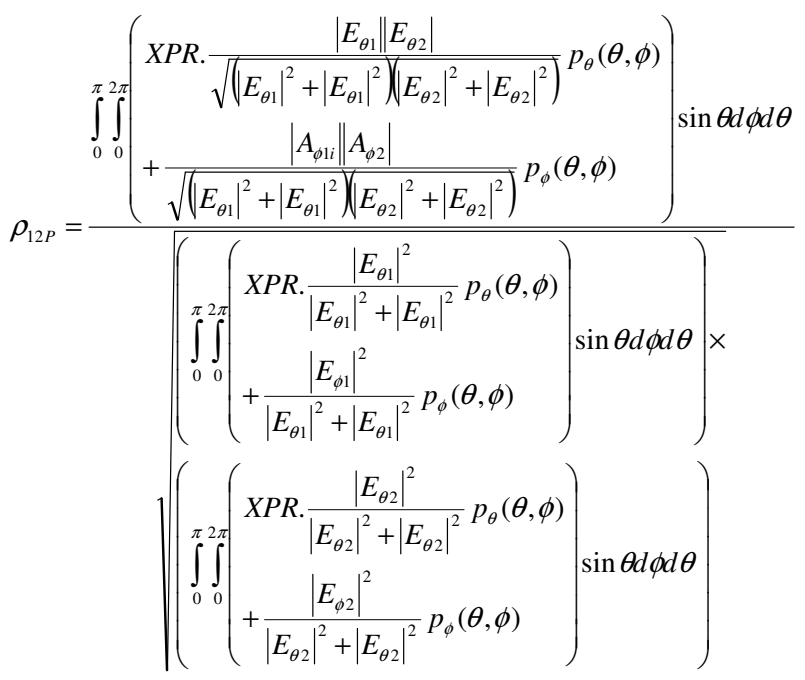

Evaluating polarisation correlation in this way provides a measure of what polarisation contribution there is to diversity. If the two antenna branches have significantly different polarisation states, then they will give a low polarisation correlation, which will in turn help achieve a low angular correlation. If the branches are copolarised, the polarisation correlation will be high (therefore little polarisation correlation) although the angular correlation could still be low. This shows how 
polarisation correlation is inherent within angular correlation at the mobile (2).

\section{Mean Effective Gain, Diversity Gain and System Gain}

Another important quantity needed to evaluate the overall diversity performance is the MEG of each antenna branch defined by the following equation (7):

MEG $=\int_{0}^{\pi} \int_{0}^{2 \pi}\left(\frac{X P R}{1+X P R} G_{\theta}(\theta, \phi) p_{\theta}(\theta, \phi)+\frac{1}{1+X P R} G_{\phi}(\theta, \phi) p_{\phi}(\theta, \phi)\right) \sin \theta d \phi d \theta$

with the same parameter definitions as in (2) plus the antenna gain functions, $G_{\theta}(\theta, \phi)$ and $G_{\phi}(\theta, \phi)$, used for the two polarisations.

Therefore, the branch power ratio can be defined as a ratio of the two mean effective gains, from which the diversity gain can be calculated (8). Further to this, it is also possible to derive the change in mean effective gain, $L$, which is the ratio of the MEG of combined IQHA branches to that of a single IQHA branch. When the branches are combined, there could be an increase in MEG (which will be advantageous to diversity) or a decrease (which will be disadvantageous).

Applying $L$ on top of the diversity gain makes it possible to determine the output gain, which is not the same as the diversity gain if there is a change in MEG. Knowing $L$ helps to determine how much of the improved output is due to diversity and how much of it is due to improved MEG. The total system gain, $\mathrm{SG}$, is related to the diversity gain, DG by:

$$
\mathrm{SG}=\mathrm{DG} \times L
$$

\section{ANTENNA MEASUREMENTS}

The two IQHA antennas were measured with elevation cuts spaced every $10^{\circ}$ in azimuth to evaluate the angular and polarisation correlation using equations (1) and (2) in discrete form. The MEG was likewise evaluated using equation (3) in discrete form.

\section{RESULTS}

The results are presented below in terms of correlation, MEG and resulting system gain. The methods of calculation are also presented to determine the MEG of and IQHA when differently combined.

\section{Correlation}

The results calculated for the adjacent and opposite element correlations are presented in TABLE 1 . As can be seen, the meandered IQHA has greater adjacent element correlation compared with the opposite correlation whereas this is reversed for the standard IQHA. However, in all cases the correlation is low enough not to degrade the diversity gain by more than $2 \mathrm{~dB}$. Therefore the branches are sufficiently decorrelated.

\begin{tabular}{|l|c|c|}
\hline & $\left|\rho_{12 A}\right|$ & $\left|\rho_{13 A}\right|$ \\
\hline Standard IQHA & 0.41 & 0.51 \\
\hline Meandered IQHA & 0.57 & 0.31 \\
\hline
\end{tabular}

TABLE 1 Magnitude of the complex angular correlation in a standard sized and meandered IQHA

For the polarisation correlation, the adjacent and opposite correlations are presented in TABLE 2. As can be seen, the polarisation correlation in all cases is high. This would be expected since the four branches are relatively co-polarised. There is a slight reduction for adjacent correlation in the case of the meandered IQHA since the meander lines do generate some cross polarisation. It can therefore be concluded that an IQHA is essentially an angular diversity system with suitably low correlation.

\begin{tabular}{|l|c|c|}
\hline & $\rho_{12 P}$ & $\rho_{13 P}$ \\
\hline Standard IQHA & 0.92 & 0.94 \\
\hline Meandered IQHA & 0.93 & 0.88 \\
\hline
\end{tabular}

TABLE 2 Polarisation correlation in a standard sized and meandered IQHA

\section{Mean Effective Gain}

The MEG needs to be calculated for a single branch (which is the same as every other branch when there is a vertical IQHA in a uniform azimuth angle of arrival) as well as the mean effective gain when different combining methods are applied. Before presenting the results, a few calculation methods need to be noted for the following combining methods:

Selection Combining. With selection combining (5), only one branch is selected at any one time. Therefore the MEG does not change, and is always the same as the MEG of a single branch if all four branches have the same MEG.

Maximum Ratio Combining (MRC). It can be proven using appropriate analysis (2), that, when MRC is applied, the MEG does not change as long as a uniform AOA in azimuth and a vertical IQHA is maintained.

Equal Gain Combining (EGC). This method is a complicated procedure. For EGC, the antennas will always have differing phase with time to apply cophasing at all the outputs. If a vertical IQHA is used in a uniform angle of arrival it can be proved that the resultant complex field pattern is (2):

$$
\begin{aligned}
& E_{\theta T}(\theta, \phi)=\frac{\left|E_{\theta 1}(\theta, \phi)\right|+\left|E_{\theta 2}(\theta, \phi)\right|+\left|E_{\theta 3}(\theta, \phi)\right|+\left|E_{\theta 4}(\theta, \phi)\right|}{2} \\
& E_{\phi T}(\theta, \phi)=\frac{\left|E_{\phi 1}(\theta, \phi)\right|+\left|E_{\phi 2}(\theta, \phi)\right|+\left|E_{\phi 3}(\theta, \phi)\right|+\left|E_{\phi 4}(\theta, \phi)\right|}{2}
\end{aligned}
$$

This is essentially a virtual $E$-field pattern that is created from the phase varying at the outputs. 
The results relevant to diversity are presented in TABLE 3 and TABLE 4. Both tables have figures for diversity gain calculated from knowing the correlation (8), the resultant change in MEG, $L$ (which is only the case for EGC) and the resulting system gain. In the cases where $L$ is $0 \mathrm{~dB}$, diversity gain and system gain are both the same. All gain values are relative to a single branch.

\begin{tabular}{|c|c|c|c|}
\hline $\begin{array}{c}\text { Combination } \\
\text { method }\end{array}$ & $\begin{array}{c}\text { Diversity } \\
\text { Gain/dB }\end{array}$ & L/dB & $\begin{array}{c}\text { System } \\
\text { Gain/dB }\end{array}$ \\
\hline $\begin{array}{c}\text { Selection } \\
\text { Combining }\end{array}$ & 13.92 & 0 & 13.92 \\
\hline EGC & 15.92 & 5.4 & 21.32 \\
\hline MRC & 16.37 & 0 & 16.37 \\
\hline
\end{tabular}

TABLE 3 Results of the diversity and system gain for a standard IQHA

\begin{tabular}{|c|c|c|c|}
\hline $\begin{array}{c}\text { Combination } \\
\text { method }\end{array}$ & $\begin{array}{c}\text { Diversity } \\
\text { Gain/dB }\end{array}$ & L/dB & $\begin{array}{c}\text { System } \\
\text { Gain/dB }\end{array}$ \\
\hline $\begin{array}{c}\text { Selection } \\
\text { Combining }\end{array}$ & 13.88 & 0 & 13.88 \\
\hline EGC & 15.88 & 5.44 & 21.32 \\
\hline MRC & 16.33 & 0 & 16.33 \\
\hline
\end{tabular}

TABLE 4 Results of the diversity and system gain for a meandered IQHA

The results show clearly that there is a significant increase in system gain for EGC, which is higher than that of the other combining methods. This is due to the high diversity gain and also the increase in MEG that adds to it. Therefore this verifies the results of the simulations and measurements in (4) and shows why EGC gives better system gain. In addition to this, Figure 3 illustrates that there is an improved MEG because EGC creates a more omni-directional field pattern than that of a single branch.

Another point to note here is that similar system gain levels can be achieved for a meandered IQHA relative to its own single branch. It should be noted, however, that the MEG of a meandered single branch is $3 \mathrm{~dB}$ less than that of a standard IQHA branch, producing an associated reduction in overall system gain.

These results only hold true on the assumption that the IQHA is vertical within an urban fading environment. For any other scenario, be that the orientation of the antenna or the propagation environment, the analysis would need to be conducted with further detail as several of the assumptions used here would not hold true.

\section{CONCLUSIONS}

The theoretical analysis of an IQHA in an urban fading environment has been presented, verifying and explaining previous measurement and simulation results. Further to this the correlation, MEG, diversity gain and system gain analysis methods have been explained. Results from this show that the IQHA is primarily an angular diversity system and provides maximum system gain when the EGC combining method is used due to the increase in MEG. A meandered IQHA has similar diversity potential to the standard IQHA although its reference branch has less efficiency.

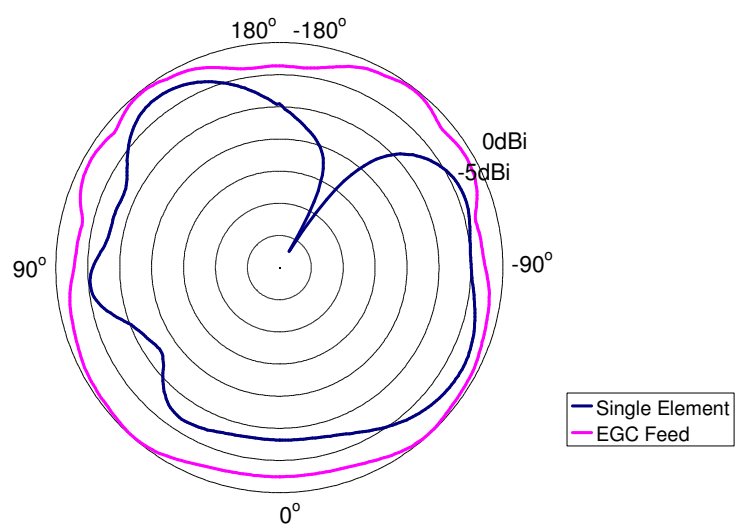

Figure 3 Comparison of a single branch, quadrature fed IQHA and EGC fed IQHA in elevation

\section{REFERENCES}

1. J. R. James, K. Fujimoto, Second Edition, 2001, "Mobile Antenna Systems Handbook", Artech House, London, UK.

2. T. W. C. Brown, 2002, “Antenna Diversity for Mobile Terminals, PhD Thesis, University of Surrey, UK.

3. S.M. Leach, A.A. Agius, S.R. Saunders, 2000, "Intelligent Quadrifilar Helix Antenna", IEE Proceedings on Microwaves, Antennas and Propagation, vol. 147, no.3, pp. 219-223.

4. S. M. Leach, 2000, “Optimum Control of HandPortable Antennas for Satellite and Terrestrial Mobile Communications", PhD Thesis, University of Surrey, UK.

5. S. R. Saunders, 1999, "Antennas and Propagation for Wireless Communication Systems”, Wiley, UK.

6. K. C. D Chew \& S.R. Saunders, "Meander Line Technique for Size Reduction of Quadrifilar Helix Antenna", accepted for publication, IEEE Antennas and Wireless Propagation Letters.

7. T. Taga, May 1990, “Analysis of Mean Effective Gain of Mobile Antennas in Land Mobile Radio Environments", IEEE Transactions on Vehicular Technology, vol. 39, no. 2, pp117-131.

8. M. Schwartz, W. R. Bennet and S. Stein, 1966, "Communication Systems and Techniques", McGraw Hill, UK. 